\section{Congrès SOFFCO}

\section{P. Topart · G. Becouarn (Présidents du congrès 2013)}

C Springer-Verlag France 2013

Cette année encore, le congrès SOFFCO a suscité plus de 120 propositions à communication, témoignant du vif intérêt pour cette manifestation.

51 communications orales et 59 affiches ont retenu l'attention du comité scientifique. Ces présentations témoignent d'une grande qualité, qu'il s'agisse de la conduite des études, de l'importance de l'expérience chirurgicale ou encore de la fiabilité du suivi, essentiel en chirurgie bariatrique.

Assez logiquement, deux grandes tendances s'affirment cette année : la diffusion du bypass gastrique avec anse en oméga (dit « mini-bypass ») et l'inexorable prédominance de la sleeve gastrectomie. Il est intéressant de constater l'insistance des rapports sur les complications, parfois dramatiques, de la chirurgie de l'obésité (y compris pour la sleeve et l'anneau gastrique) ainsi que sur le problème des échecs de perte pondérale sur le long terme, ce qui témoigne de la grande honnêteté des participants au congrès de la SOFFCO.

Tout cela s'avère fort prometteur en vue de passionnants débats.

Rendez-vous donc le 6 juin à Angers.

Cordialement

P. Topart $(\bowtie) \cdot$ G. Becouarn

e-mail : ptopart@gmail.com 